\title{
Die transseptale Punktion - Schritt für Schritt
}

Arian Sultan

\author{
Die transseptale Punktion ist zu einem Routineeingriff in der invasiven Elektrophy- \\ siologie und Kardiologie geworden. Sie dient primär dem Zugang zum linken Vorhof, \\ zum Beispiel für linksatriale Ablationen, LAA-Occluderimplantationen oder den \\ perkutanen Mitralklappeneingriff. Unter Einhaltung eines strukturierten Ablaufs \\ ist die transseptale Punktion sicher, komplikationsarm und ausschließlich unter \\ Durchleuchtung durchführbar.
}

\section{Allgemeines}

Die ersten Punktionen erfolgten bereits in den 1950er Jahren [1 - 3]. Durch die Weiterentwicklung der invasiven Elektrophysiologie und Kardiologie hat die Anzahl der transseptalen Punktionen (TSP) deutlich zugenommen. Die TSP ermöglicht über das venöse System einen Zugang sowohl zum linken Vorhof (LA) als auch zum linken Ventrikel (LV). Dieser Zugangsweg ist essenziell, um z. B. eine Pulmonalvenenisolation oder eine perkutane Mitralklappenintervention durchführen zu können. Aber auch für den Zugang zum LV z.B. nach Aortenklappenersatz mit einer mechanischen Prothese benötigt man die TSP. In der Anfangsphase der Durchführung kann eine begleitende transösophageale Echokardiografie zur besseren Lokalisation der Punktionsstelle hilfreich sein.

\section{Durchführung}

Abhängig vom geplanten Eingriff werden 1-2 lange Punktionsschleusen (z.B. SLO ${ }^{\mathrm{TM}} 10 \mathrm{~F}$ Fast-Cath ${ }^{\mathrm{TM}}$ ) über eine venöse Leistenpunktion bis in die V. cava superior vorgebracht.

Anschließend wird die Brockenbrough-Punktionsnadel (BRK ${ }^{\mathrm{TM}}, \mathrm{BRK}-1^{\mathrm{TM}}$, BRK-2 ${ }^{\mathrm{TM}}$, BRK-XS'M ${ }^{\mathrm{TM}}$ ) mit ihrer Spitze bis ca. $2 \mathrm{~cm}$ hinter das Schleusenende in die Schleuse eingeführt. Diese Position muss manuell gesichert werden, damit die Nadel beim Zurückziehen nicht heraustritt. Unter geringer Kontrastmittelgabe (optional) und unter Durchleuchtung (Strahlengang a.-p. oder LAO) gleitet die Schleuse unter vorsichtigem Rückzug am Septum entlang nach kaudal. Dabei ist sie leicht nach links-posterior („16-Uhr-Position“) ausgerichtet und die Nadel noch nicht ausgefahren.
Erreicht man die Fossa ovalis, kommt es idealerweise zu einem sichtbaren Sprung der Schleuse in die Fossa ovalis. Fährt man nun die Nadel unter Kontrastmittelgabe aus der Schleuse heraus, kann ein „Tenting“ des Septums sichtbar sein. Hier kann nun die Punktion des intraatrialen Septums sicher erfolgen.

\section{PRAXISTIPP}

Sollte die Passage erschwert sein, ist die Position möglicherweise zu weit anterior. Durch eine leichte Drehung der Schleuse samt Nadel nach posterior (im Uhrzeigersinn) kann eine Fehlpunktion der Aorta vermieden werden.

Ist die Punktion adäquat erfolgt, sollte nach Übertritt der Schleuse in den linken Vorhof Blut aspirabel sein. Verifizierbar ist dies, indem sich der linke Vorhof nach Gabe von ca. 7-10 ml Kontrastmittel (KM) anfärben lässt.

Wenn sichergestellt ist, dass sich Nadel und Schleuse im LA befinden, sollte die Nadel zügig entfernt werden und nach neuerlicher Aspiration der Schleuse ein Draht, Katheter oder Device in den LA eingeführt werden. Von diesem Zeitpunkt an muss eine effektive Antikoagulation des Patienten erfolgen, üblicherweise durch gewichtsadaptierte Gabe von unfraktioniertem Heparin. Die Dosierung der weiteren Heparingaben hängt dann von der ACT (Activated Clotting Time) ab.

Cave

Da der Blutdruck im LA sehr niedrig ist und sogar kurzeitig negative Bereiche aufweisen kann, sollte das akzidentelle Ansaugen von Luft vermieden werden, indem das Schleusenende immer verschlossen ist oder möglichst tief gehalten wird. 


\section{Schritt 1}

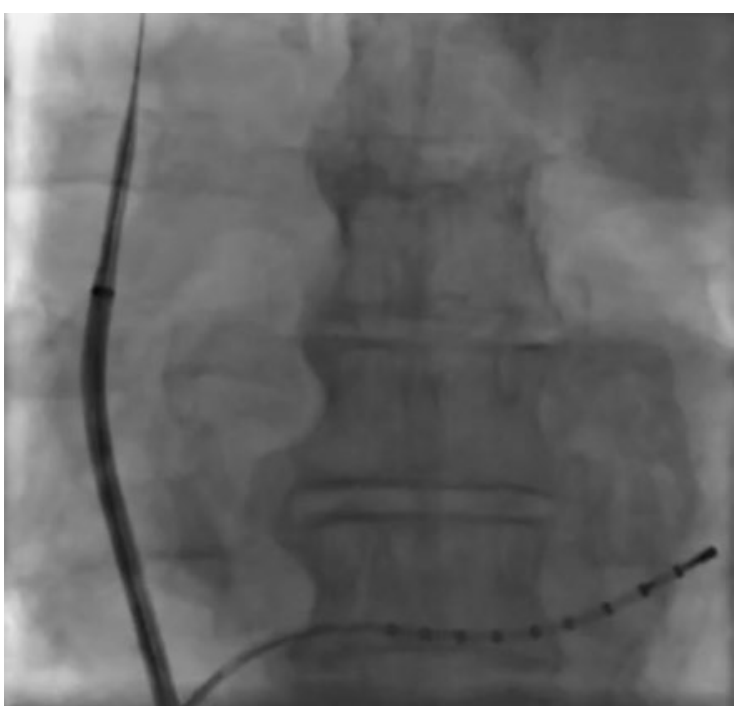

- Abb. 1 Führen Sie die Punktionsschleuse (hier SL-0) mithilfe eines langen Drahtes vor bis in die V. cava superior. Sichtbarer Diagnostikkatheter im Koronarvenensinus bei geplanter Pulmonalvenenisolation.

\section{Schritt 2}

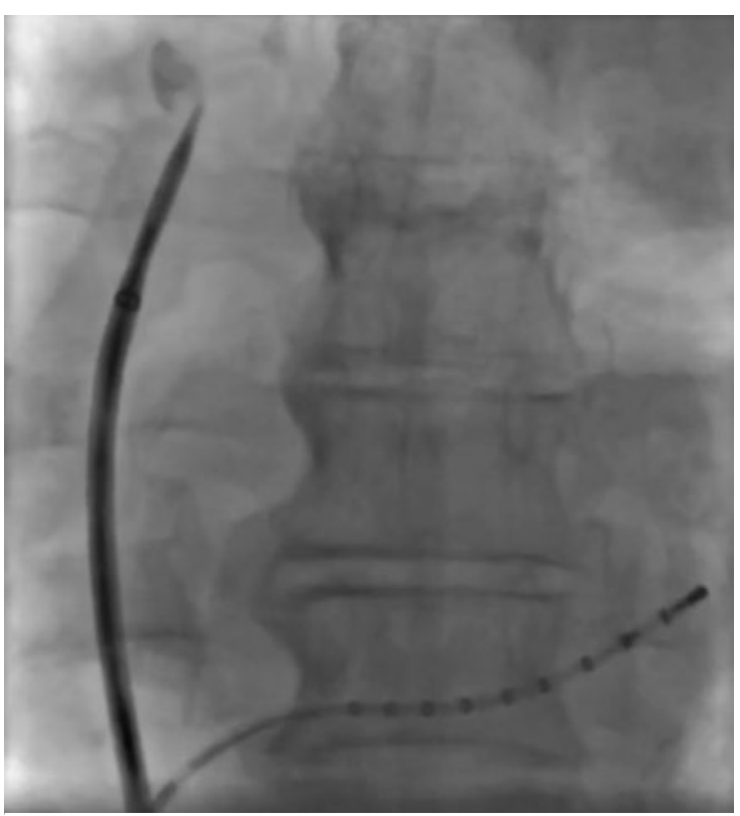

- Abb. 2 Nach Entfernen des Drahtes und Spülen der Schleuse wird die transseptale Nadel eingeführt. Diese sollte jedoch die Schleuse noch nicht verlassen (Ende ca. $2 \mathrm{~cm}$ vor Schleusenspitze). Über die transseptale Nadel kann Kontrastmittel appliziert werden.

\section{Schritt 3}

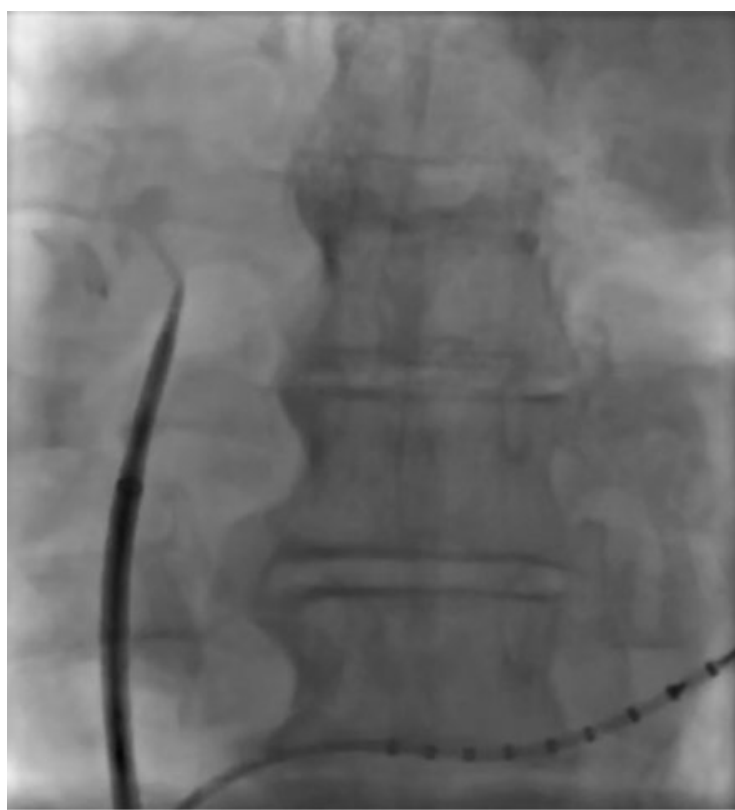

- Abb. 3 Ziehen Sie jetzt die Schleuse unter leichter Kontrastmittelgabe über die transseptale Nadel nach kaudal. Dabei wird die Schleuse leicht nach posterior rotiert („16-Uhr-Position“) gehalten.

\section{PRAXISTIPP}

Wichtig ist, dass Schleuse und transseptale Nadel gleich ausgerichtet sind: so ist deren Führung leichter und risikoärmer. 


\section{Schritt 4}

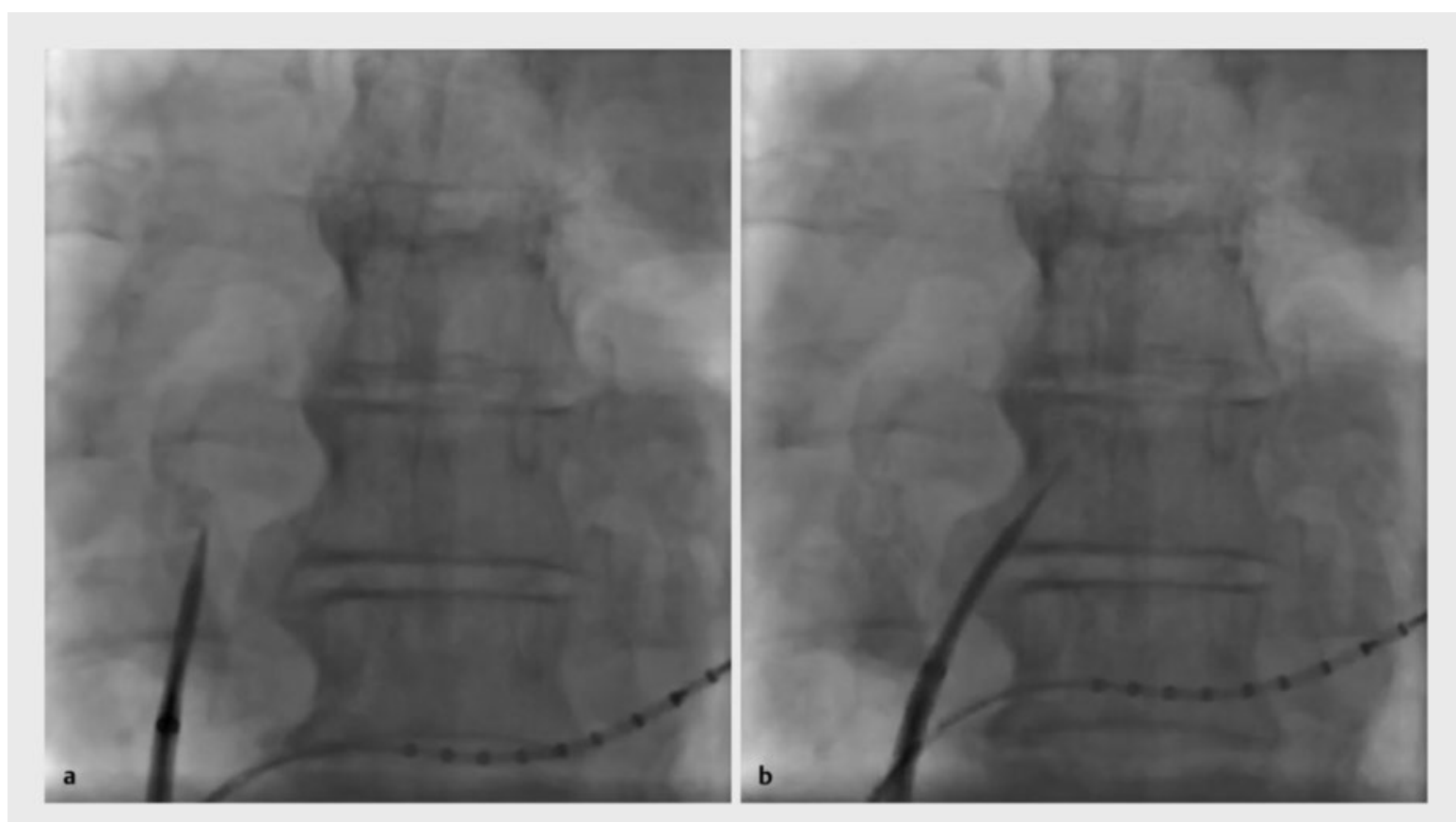

- Abb. 4 Bei Erreichen der Fossa ovalis kommt es idealerweise zu einem sichtbaren Sprung in die Fossa ovalis. Als weitere Orientierung kann der Abstand zum Ostium des Koronarvenensinus dienen: Die Fossa ovalis befindet sich ca. einen Wirbelkörper über dem Ostium des Koronarvenensinus.

\section{Schritt 5}

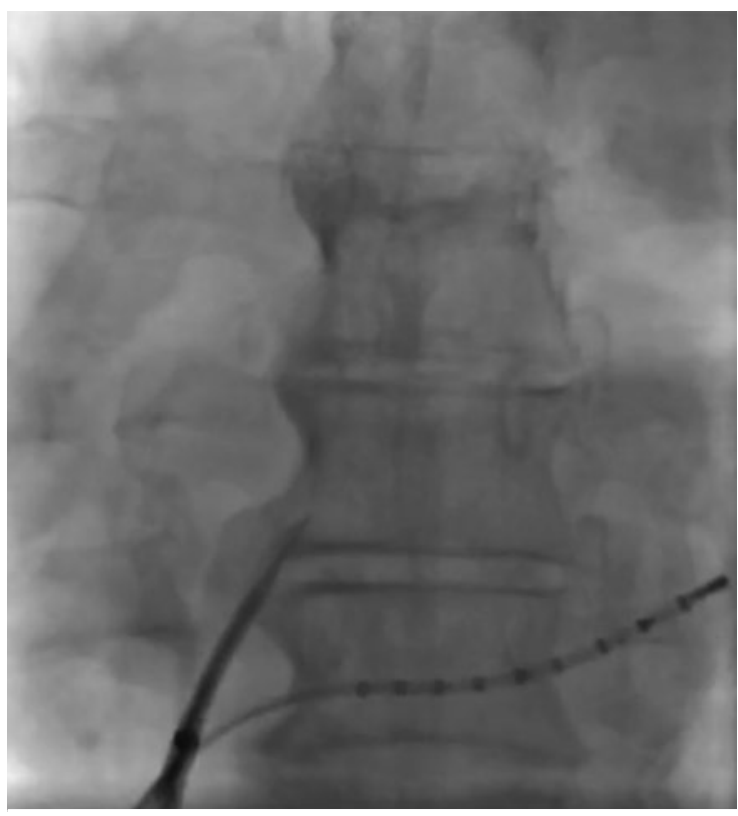

- Abb. 5 Jetzt wird die transseptale Nadel unter Kontrastmittelgabe ausgefahren. Dabei entsteht mitunter ein „Tenting“ („Aufspannen“) des Septums, bevor die Nadel das Septum passiert.

\section{Schritt 6}

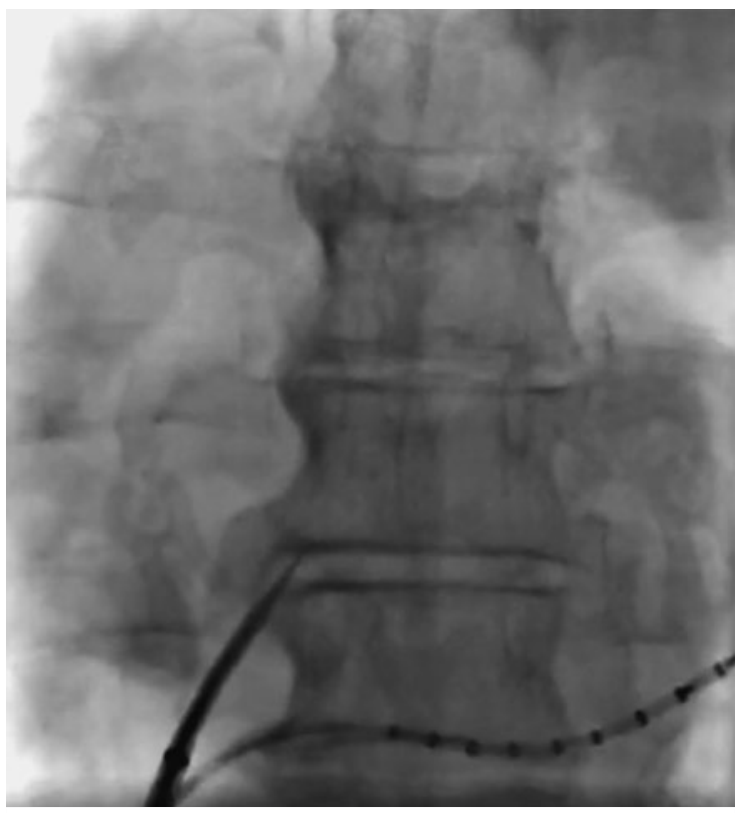

- Abb. 6 Nach Passage des Septums mit der transseptalen Nadel sollte Blut aspirabel sein. Anschließend erfolgt eine Verifizierung der erfolgreichen Punktion mittels Kontrastmittelgabe. 


\section{Schritt 7}

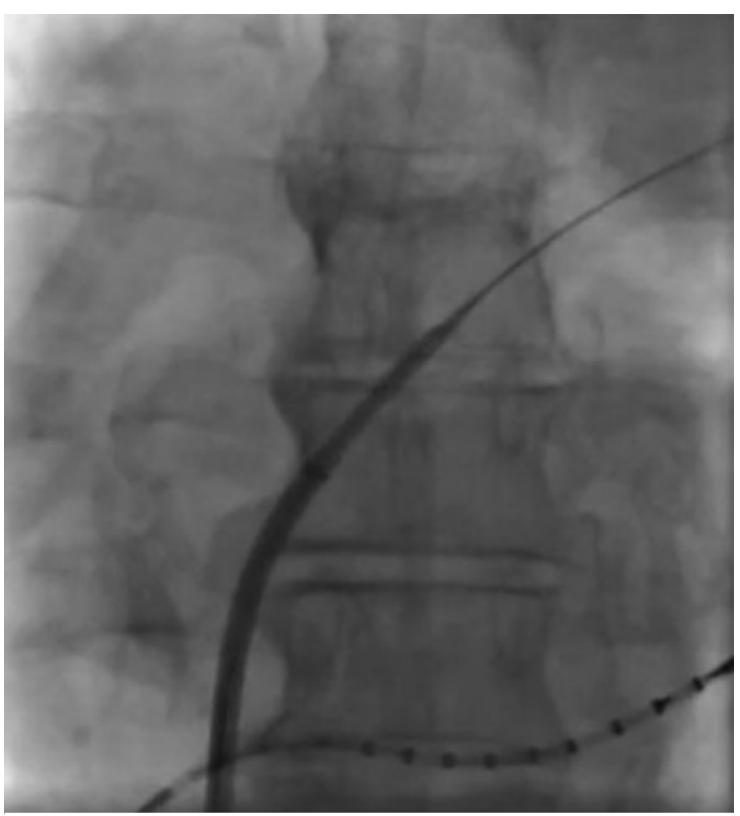

Abb. 7 Nach erfolgreicher Punktion und minimalem Vorbringen der Schleuse (damit sich nicht nur die Nadelspitze im linken Vorhof befindet), kann die transseptale Nadel entfernt werden. Umgehend sollte jetzt ein Draht über die Schleuse eingeführt werden. Über den Draht kann die Schleuse weiter vorgebracht und somit das Septum aufbougiert werden. Vor diesem Schritt sollte jedoch sichergestellt werden, dass der Draht den Herzschatten verlässt und somit in der Pulmonalvene liegt (siehe Abb. 8).

\section{Cave}

$A b$ jetzt ist eine effektive Antikoagulation - üblicherweise mit Heparin - obligat, um eine arterielle Thromboembolie zu vermeiden!

\section{Schritt 8}

\section{Merke}

Um eine akzidentielle Luftembolie zu vermeiden, sollte vor Kontrastmittelgabe noch einmal Blut über die Schleuse aspiriert werden. Ebenfalls sollte die Kontrastmittelspritze niemals vollständig appliziert werden (erneute Luftembolie-Gefahr).

- Abb. 8 Nach der Aufbougierung wird die Schleuse über den im linken Vorhof liegenden Draht in den linken Vorhof vorgebracht. Anschließend entfernt man den Draht und den Mandrin der Schleuse. Nun kann über die Schleuse eine Angiografie der Pulmonalvenen erfolgen (jede Seite einzeln oder als Gesamtaufnahme unter Adenosingabe).
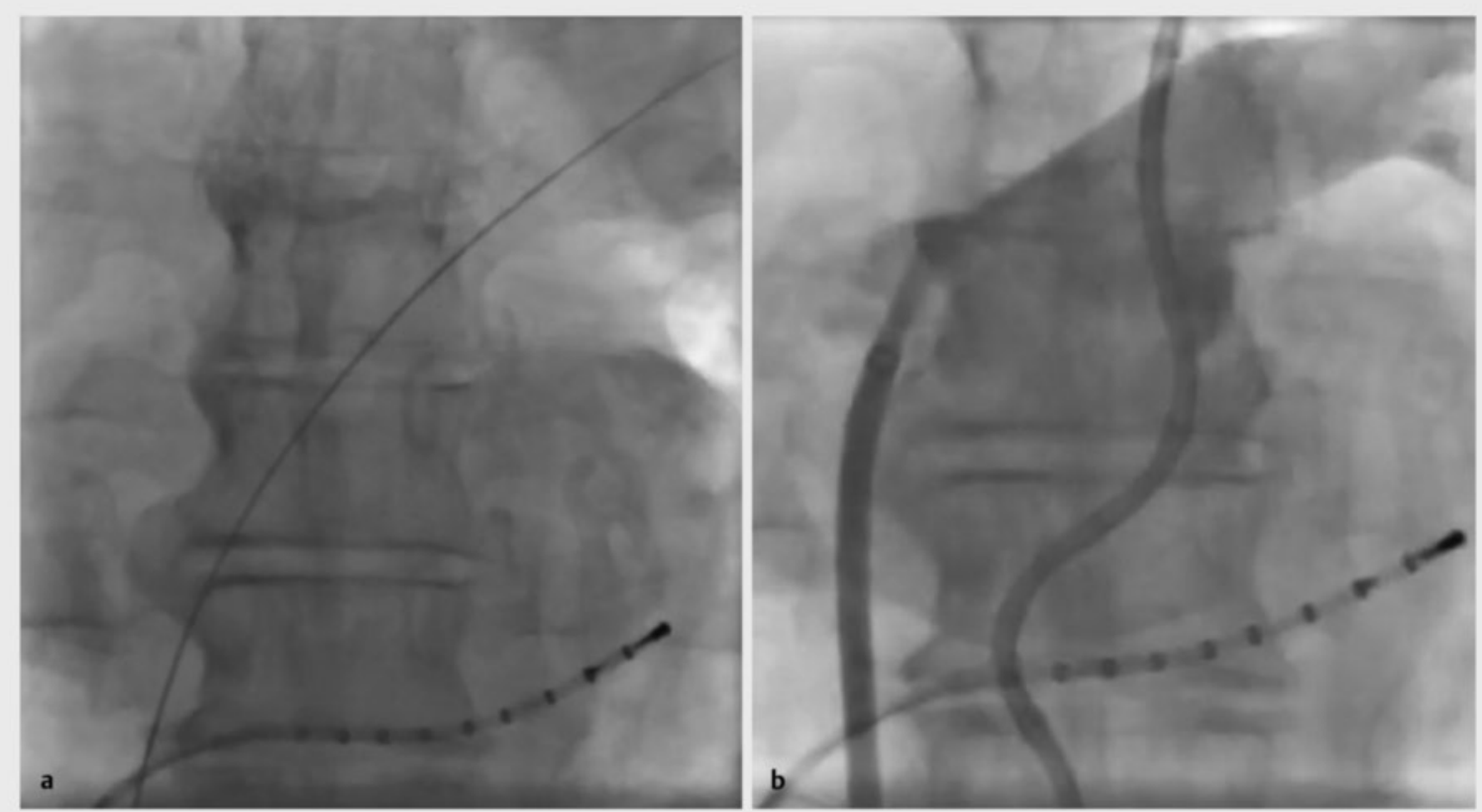


\section{Komplikationen}

Die Komplikationsrate der TSP liegt in der Regel bei $<1 \%$ [4]. Neben einer versehentlichen Punktion nach epikardial ist die Fehlpunktion der Aorta ascendens mit eventueller Perikardtamponade die gefürchtetste Komplikation. Verschiedene Angulationen (z. B. a.-p. vs. LAO) sowie optional die Verwendung der transösophagealen Echokardiografie in schwierigen Fällen vor der Punktion können das Eingriffsrisiko verringern.

\section{Interessenkonflikt}

Die Autoren geben an, dass kein Interessenkonflikt besteht.

Autorinnen/Autoren

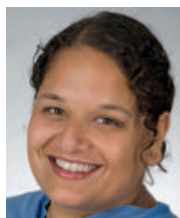

\section{Arian Sultan}

Dr. med. Arian Sultan ist Oberärztin in der Abteilung für Elektrophysiologie an der III. Medizinischen Klinik des Herzzentrums der Universität zu Köln. Ihr Schwerpunkt ist die Elektrophysiologie.

[1] Ross I jr., Braunwald E, Morrow AG. Left heart catheterization by the transseptal route: a description of the technic and its applications. Circulation 1960; 22: 8

[2] Brockenbrough EC, Braunwald E, Ross J jr. Transseptal left heart catheterization. A review of 450 studies and description of an improved technic. Circulation 1962; 25: 15-21

[3] Mullins CE. Transseptal left heart catheterization: experience with a new technique in 520 pediatric and adult patients. Pediatr Cardiol 1983; 4: 239-245

[4] Manolis AS. Transseptal access to the left atrium: Tips and tricks to keep it safe derived from single operator experience and review of the literature. Curr Cardiol Rev 2017; 13: 305318

\section{Bibliografie}

DOI https://doi.org/10.1055/a-0674-6845

Kardiologie up2date 2019; 15: 95-99

(c) Georg Thieme Verlag KG Stuttgart · New York ISSN 1611-6534

Korrespondenzadresse

Dr. med. Arian Sultan

Abteilung für Elektrophysiologie

III. Medizinische Klinik

Herzzentrum der Universität zu Köln

Kerpener Str. 62

50937 Köln

arian.sultan@uk-koeln.de 